\title{
Reexamining estrogen
}

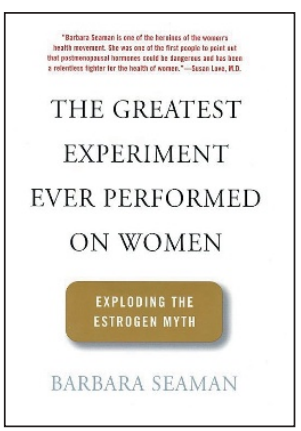

\section{The Greatest Experiment Ever Performed on Women: Exploding the Estrogen Myth}

\begin{tabular}{l} 
By Barbara Seaman \\
\hline Hyperion Books, 2003 \\
332 pp. hardcover, $\$ 24.95$ \\
ISBN 0-7868-6853-8 \\
Reviewed by \\
C Dominique Toran-Allerand
\end{tabular}

This book provides an extensive history of hormonal treatment of postmenopausal women, the dangers of those treatments, the basic scientists who developed the hormones, the pharmaceutical industry whose sole interest was in marketing the hormones, and the physicians interested in financial gain. All this forms the backdrop for Seaman's thesis: estrogen treatment of women is generally unwarranted and harmful. The author describes how large numbers of women became unsuspecting guinea pigs for unscrupulous doctors and drug companies who foisted estrogen treatment upon them. She uses the recent Women's Health Initiative (WHI) studies to support her view that menopause does not require hormonal treatment, as it is "a natural part of life and not a disease."

It is unfortunate that the author has chosen to perpetuate the negative media coverage and to present the results of the WHI studies so uncritically. What seems to be totally lacking from this book is an understanding of the biology of estrogen and progesterone, consideration of the limitations of the WHI study designs, and an appreciation of the nature of and reasons for the negative results.

Most of this book focuses on the harmful effects of the most commonly used but worst hormones available: the conjugated equine estrogens (Premarin), the synthetic progestin (Provera; medroxyprogesterone acetate), or their combination (PremPro). The WHI studies were designed to address whether Premarin or PremPro, given concurrently and continuously for prolonged periods of time (5+ years), would be beneficial for postmenopausal women. The PremPro arm of the study was abruptly terminated after 5.2 years because of increased incidence of breast cancer, heart attacks, strokes, blood clots and pulmonary emboli. There were, on the other hand, significantly fewer cases of colon cancer and bone fractures. While these studies were successful in discrediting this form of treatment, the results have been so greatly overinterpreted and applied so generally as to discredit all hormone replacement, an issue never addressed by the WHI.

C. Dominique Toran-Allerand is in the Departments of Anatomy \& Cell Biology and Neurology, and Centers for Neurobiology \& Behavior and Reproductive Sciences, Columbia University College of Physicians \& Surgeons, New York, New York 10032, USA.

e-mail: cd2@columbia.edu
Unfortunately, Seaman is drawn in by this generality. She focuses on the carcinogenic potential of estrogen for the breast, but does not consider the more likely culprit-Provera. Provera is a synthetic analog of progesterone, and although estrogen (estradiol) and progesterone normally act synergistically, Provera actually blocks many of the beneficial actions of estrogen. Moreover, although Provera, like progesterone, stimulates cell division in the breast, Provera does so more strongly (by binding to glucocorticoid as well as progesterone receptors). The author ignores the ongoing estrogen-only arm of the WHI study, which has not shown the increase in breast cancer rates that led to the abrupt termination of the PremPro arm. Concurrent and continuous exposure to nonhuman estrogens and progestins for prolonged periods, as in the WHI studies, is very different from the normal sequential and cyclical physiological pattern of exposure and can lead to nonphysiological responses.

This book also fails to consider the importance of estrogen and progesterone for the brain. Estradiol influences higher cognitive functions, mood, pain, fine motor skills, hot flashes, sleep and susceptibility to seizures. Estradiol also has neuroprotective effects that shield the brain from damage caused by ischemic stroke and Alzheimer and Parkinson diseases. Prolonged, continuous exposure to estrogen downregulates estrogen receptor levels, however, and diminishes the ability of neurons to respond to estrogen. Uninterrupted exposure to estrogen over years, as in the WHI study, represents the most catastrophic condition imaginable for normally aging estrogen target neurons, particularly neurons already at risk for Alzheimer disease. Discontinuing estrogen for a few days in a cyclical manner, as normally occurs during the female reproductive cycle, enables upregulation of estrogen receptor levels, allowing neurons to become more responsive to estrogen again.

Progesterone and its metabolites are also neuroprotective for the brain, and have been shown to have sedative, hypnotic, anesthetic, anxietyreducing, sleep-modulating, anticonvulsant, antidepressant and antipsychotic effects. In contrast, not only is Provera an ineffective neuroprotectant, but it actually antagonizes estrogen-induced neuroprotection and exhibits none of progesterone's beneficial properties.

This book perpetuates the dogma, uncritically championed by the WHI and earlier studies, that estrogen has no beneficial effects on the heart or in Alzheimer disease, and does not consider the nonphysiological design of these studies and how hormones were used.

On the plus side, the appendix contains a helpful review of all available estrogen, progestin and androgen preparations as well as their side effects. Also included are alternative treatments for menopause: various herbal and plant supplements, in particular the phytoestrogens (black cohosh, soy and red clover) and the more recently introduced serotonin reuptake inhibitors (SSRIs; Prozac, Paxil and Zoloft). Phytoestrogens and SSRIs are used primarily for treating hot flashes, but their benefits are really not clear.

Seaman has failed to provide a critical presentation of the state of our understanding of hormone replacement therapy in disease prevention. Indeed, the therapeutic potential of estradiol and progesterone has not yet received a proper scientific evaluation. It is imperative that the basis for the negative results of the WHI studies-namely the failure to take into account the biology of these hormones and their receptors-be properly and fully addressed in the future. 
ERRATUM: Reexamining estrogen

C Dominique Toran-Allerand

Nat. Med. 10, 121 (2004)

The author's e-mail address was printed incorrectly. The correct address is cdt2@columbia.edu.

CORRIGENDUM: Hypothalamic gray matter changes in narcoleptic patients

B Draganski, P Geisler, G Hajak, G Schuierer, U Bogdahn, J Winkler \& A May

Nat. Med. 8, 1186-1188 (2002)

Because of space constraints, a reference was omitted from the text. The following sentence should have appeared after sentence 5 in paragraph 3: "However, Kaufmann et al. have described changes in gray matter structures in 12 narcoleptic patients ${ }^{10}$." Subsequent references should be renumbered accordingly. The new reference 10 should be as follows:

10. Kaufmann, C., Schuld, A., Pollmacher, T. \& Auer, D.P. Reduced cortical gray matter in narcolepsy: preliminary findings with voxel-based morphometry. Neurology 58, $1852-1855$ (2002).

CORRIGENDUM: Differentiating the pathologies of cerebral malaria by postmortem parasite counts

Terrie E Taylor, Wenjiang Fu, Richard A Carr, Rich O Whitten, Jeffrey G Mueller, Nedson M Fosiko, Susan Lewallen, N George Liomba \& Malcolm E Molyneux

Nat. Med. 10, 143-145 (2004)

The name of author Jeffrey S Mueller was spelled incorrectly. 